\title{
Non-BPS Dirichlet branes
}

\author{
Oren Bergman $\dagger$ and Matthias R Gaberdiel $\ddagger$ \\ $\dagger$ California Institute of Technology, Pasadena, CA 91125, USA \\ \$Department of Applied Mathematics and Theoretical Physics, University of Cambridge, \\ Silver Street, Cambridge CB3 9EW, UK
}

Received 26 August 1999

\begin{abstract}
A brief introduction to the boundary state approach to Dirichlet branes is given. The example of the non-BPS D-string of type IIA on K3 is analysed in some detail, and its dual heterotic state is identified.

PACS numbers: $1125,1125 \mathrm{~S}$
\end{abstract}

\section{Introduction}

Over the past year, Dirichlet branes (D-branes) which do not preserve supersymmetry, and therefore do not saturate the Bogomolnyi-Prasad-Sommerfield (BPS) bound, have attracted a fair amount of attention $[1,2]$. There exist in essence two different approaches to constructing and analysing these states. In one approach that has been pioneered by Sen [3-7] the nonBPS D-branes are constructed as bound states of brane-anti-brane pairs. This construction has been interpreted in terms of K-theory by Witten [8], and this has opened the way for a more mathematical treatment of D-branes [9-11]. In the other approach, D-branes (and, in particular, non-BPS D-branes) are described as coherent states in the closed string theory that satisfy a number of consistency conditions [12-17]. This approach will be explained in more detail in the next section.

The motivation for studying non-BPS D-branes is threefold. First, in order to understand the strong-weak coupling dualities of supersymmetric string theories in more detail, it is important to analyse how these dualities act on states that are not BPS saturated. Since the dualities typically map perturbative states to non-perturbative (D-brane-type) states, we will naturally encounter non-BPS D-branes in these considerations. Similarly, if we are to make progress in analysing the possible dualities of theories without supersymmetry $\dagger$, we have to develop techniques to treat Dirichlet branes without supersymmetry. Secondly, since the field theories describing the low-energy dynamics of non-BPS D-branes are nonsupersymmetric gauge theories, configurations of non-BPS D-branes might be useful for studying non-perturbative aspects of the corresponding field theories. Finally, non-BPS Dbranes offer the intriguing possibility of string compactifications in which supersymmetry is preserved in the bulk but broken on the brane.

This paper is based primarily on [21]; more details about the boundary state approach can also be found in $[16,19]$.

$\dagger$ Some progress in this direction has been made recently in [16, 18-20]. 


\section{Dirichlet branes from boundary states}

A D-brane can be represented by a coherent (boundary) state of the closed string theory that describes the D-brane as a source for closed strings. Such a state describes a submanifold on which open strings can end provided that it satisfies

$$
\begin{array}{ll}
\left(\partial X^{i}-\bar{\partial} X^{i}\right)|B\rangle=0 & i \text { Neumann direction, } \\
\left(\partial X^{I}+\bar{\partial} X^{I}\right)|B\rangle=0 & I \text { Dirichlet direction, }
\end{array}
$$

where $X^{\mu}$ denotes the coordinate field. In the following we shall always work in the lightcone NS-R formalism. The directions $\mu=0,1$ will be taken to be Dirichlet, so the states are actually related by a double Wick rotation to normal D-branes [15].

For a $\mathrm{D} p$-brane, the above conditions can be rewritten in terms of modes as

$$
\begin{aligned}
& \left.\begin{array}{l}
\left(\alpha_{n}^{\mu}-\widetilde{\alpha}_{-n}^{\mu}\right)|B p, \eta\rangle=0 \\
\left(\psi_{r}^{\mu}-\mathrm{i} \eta \widetilde{\psi}_{-r}^{\mu}\right)|B p, \eta\rangle=0
\end{array}\right\} \quad \mu=2, \ldots, p+2 \\
& \left.\begin{array}{l}
\left(\alpha_{n}^{\mu}+\widetilde{\alpha}_{-n}^{\mu}\right)|B p, \eta\rangle=0 \\
\left(\psi_{r}^{\mu}+\mathrm{i} \eta \widetilde{\psi}_{-r}^{\mu}\right)|B p, \eta\rangle=0
\end{array}\right\} \quad \mu=p+3, \ldots, 9,
\end{aligned}
$$

where $\alpha_{n}^{\mu}, \widetilde{\alpha}_{n}^{\mu}$ and $\psi_{r}^{\mu}, \widetilde{\psi}_{r}^{\mu}$ are the left- and right-moving modes of the bosonic and fermionic fields, respectively, and $\eta= \pm$ describes the two different spin structures. For each choice of $\eta$ and in each left-right-symmetric sector of the theory (i.e. in the NS-NS and the R-R sectors), a unique solution (up to normalization) to these equations exists

$$
\begin{aligned}
|B p, \eta\rangle=\exp & \left\{\sum_{n>0}\left[-\frac{1}{n} \sum_{\mu=2}^{p+2} \alpha_{-n}^{\mu} \widetilde{\alpha}_{-n}^{\mu}+\frac{1}{n} \sum_{\mu=p+3}^{9} \alpha_{-n}^{\mu} \widetilde{\alpha}_{-n}^{\mu}\right]\right. \\
& \left.+\mathrm{i} \eta \sum_{r>0}\left[-\sum_{\mu=2}^{p+2} \psi_{-r}^{\mu} \widetilde{\psi}_{-r}^{\mu}+\sum_{\mu=p+3}^{9} \psi_{-r}^{\mu} \widetilde{\psi}_{-r}^{\mu}\right]\right\}|B p, \eta\rangle^{(0)} .
\end{aligned}
$$

Here $r$ is half-integer in the NS-NS sector and integer in the R-R sector. The ground state $|B p, \eta\rangle^{(0)}$ is the tachyonic ground state in the case of the NS-NS sector, and it is uniquely determined by the condition (2) and (3) with $r=0$ in the R-R sector.

Given two coherent boundary states, we can calculate the closed string tree amplitude that describes the exchange of closed string states between the two boundary states. Because of worldsheet duality, this amplitude can be reinterpreted as a one-loop open string vacuum amplitude. The actual D-brane state is a certain linear combination of these boundary states in different sectors and with different spin structures [16]; this linear combination is characterized by the condition that it satisfies:

(a) It is a physical state of the closed string theory, i.e. it is GSO invariant, and invariant under orbifold and orientifold projections where appropriate.

(b) The open string amplitude obtained by worldsheet duality from the closed string exchange between any two D-branes constitutes an open string partition function, i.e. it corresponds to a trace over a set of open string states of the open string time-evolution operator.

(c) The open strings that are introduced in this way have consistent string field interactions with the original closed strings.

In this paper we shall be mainly interested in stable D-branes; this requires, in particular, that the spectrum of open strings that begin and end on the same D-brane does not contain a tachyon. 
The conditions that we have outlined above are intrinsic consistency conditions of the interacting string (field) theory; in particular, they are more fundamental than spacetime supersymmetry, and also apply in cases where spacetime supersymmetry is broken or absent.

\subsection{An example: type IIA and IIB}

In order to demonstrate that these conditions capture the essence of D-branes, let us analyse, as an example, the case of type IIA/IIB string theory. In the NS-NS sector, a GSO-invariant boundary state exists for all $p$,

$$
|B p\rangle_{N S N S}=\left(|B p,+\rangle_{N S N S}-|B p,-\rangle_{N S N S}\right),
$$

but it does not describe a stable D-brane by itself since the open string that begins and ends on $|B p\rangle_{N S N S}$ consists of an unprojected NS and R sector, and therefore contains a tachyon in its spectrum. In fact (4) describes the unstable D $p$-brane for $p$ odd (even) in type IIA (IIB) that was described in [1]; for $p=9$, this also coincides with the unstable D9-brane of type IIA that was mentioned in [9].

In order to obtain a stable D-brane, we have to add to (4) a boundary state in the R-R sector; the only potentially GSO-invariant state is of the form

$$
|B p\rangle_{R R}=\left(|B p,+\rangle_{R R}+|B p,-\rangle_{R R}\right),
$$

and it is actually GSO-invariant if $p$ is even (odd) in the case of type IIA (IIB). If this is the case, we can find a suitable linear combination $\dagger$

$$
|D p\rangle=\mathcal{N}_{N S N S}|B p\rangle_{N S N S} \pm \mathcal{N}_{R R}|B p\rangle_{R R},
$$

whose corresponding open string spectrum consists of the GSO-projected NS and R sector. Thus, we have shown that the only stable D-branes in type IIA and IIB are the familiar BPS D-branes.

\section{Non-BPS states in heterotic-type II duality}

Many string theories contain states that are not BPS-saturated but are stable due to the fact that they are the lightest states of a given charge [3]. Because of their stability, these states must also be present in the dual theory.

One particularly interesting example where both theories can be analysed in detail is the duality between the heterotic string on $T^{4}$ and type IIA on K3 [22]. We shall, in particular, consider the orbifold point of $\mathrm{K} 3$, where $\mathrm{K} 3=T^{4} / \mathbb{Z}_{2}$, since one can then easily define boundary states in the IIA theory. The sequence of dualities relating the two theories is given by

$$
\text { het } \quad T^{4} \stackrel{S}{\longrightarrow} \text { I } \quad T^{4} \stackrel{T^{4}}{\longrightarrow} \text { IIB } \quad T^{4} / \mathbb{Z}_{2}^{\prime} \stackrel{S}{\longrightarrow} \text { IIB } \quad T^{4} / \mathbb{Z}_{2}^{\prime \prime} \stackrel{T}{\longrightarrow} \text { IIA } \quad T^{4} / \mathbb{Z}_{2},
$$

where the various $\mathbb{Z}_{2}$ groups are

$$
\mathbb{Z}_{2}^{\prime}=\left(1, \Omega \mathcal{I}_{4}\right) \quad \mathbb{Z}_{2}^{\prime \prime}=\left(1,(-1)^{F_{L}} \mathcal{I}_{4}\right) \quad \mathbb{Z}_{2}=\left(1, \mathcal{I}_{4}\right)
$$

Here $\mathcal{I}_{4}$ reflects all four compact directions, $\Omega$ reverses worldsheet parity and $F_{L}$ is the leftmoving part of the spacetime fermion number. It follows from (7) that the parameters of the

$\uparrow$ The sign in (6) distinguishes a brane from an anti-brane. 
two theories are related as

$$
\begin{aligned}
& g_{A}=g_{h}^{-1} R_{h 4} V_{h}^{1 / 2} \\
& R_{A j}=\frac{2}{R_{h j}} V_{h}^{1 / 2} \quad \text { for } \quad j \neq 4 \\
& R_{A 4}=\frac{1}{2} V_{h}^{-1 / 2} R_{h 4},
\end{aligned}
$$

where $g_{A}, R_{A i}$ and $g_{h}, R_{h i}$ are the coupling constant and the radii of the IIA and the heterotic theory, respectively, and $V_{h}=R_{h 1} R_{h 2} R_{h 3} R_{h 4}$.

At the orbifold point, the gauge group of the IIA theory is $U(1)^{24} ; 16$ of these $U(1)$ 's arise from the 16 twisted R-R sectors that are associated with the fixed planes of the orbifold, and the remaining eight $U(1)$ 's come from the 1 -form and the 3 -form in ten dimensions. In the dual heterotic theory (that we shall take to be the $\operatorname{Spin}(32) / \mathbb{Z}_{2}$ theory), we also have to have an Abelian gauge group, and this requires that appropriate Wilson lines are turned on

$$
\begin{aligned}
& A^{1}=\left(\left(\frac{1}{2}\right)^{8}, 0^{8}\right) \\
& A^{2}=\left(\left(\frac{1}{2}\right)^{4}, 0^{4},\left(\frac{1}{2}\right)^{4}, 0^{4}\right) \\
& A^{3}=\left(\left(\frac{1}{2}\right)^{2}, 0^{2},\left(\frac{1}{2}\right)^{2}, 0^{2},\left(\frac{1}{2}\right)^{2}, 0^{2},\left(\frac{1}{2}\right)^{2}, 0^{2}\right) \\
& A^{4}=\left(\frac{1}{2}, 0, \frac{1}{2}, 0, \frac{1}{2}, 0, \frac{1}{2}, 0, \frac{1}{2}, 0, \frac{1}{2}, 0, \frac{1}{2}, 0, \frac{1}{2}, 0\right) .
\end{aligned}
$$

These break the gauge group $S O(32)$ to $S O(2)^{16} \sim U(1)^{16}$. The actual form of the Wilson lines can be confirmed, a posteriori, by comparing the masses of the various BPS states. Let us analyse some of them in turn.

Bulk BPS D-particles. The type IIA orbifold possesses a bulk D-particle, which corresponds to a type IIA D-particle together with its image under $\mathbb{Z}_{2}$. This state carries unit charge under the ten-dimensional R-R 1-form $C_{R R}^{(1)}$, and is described by

$$
|D 0 ; \epsilon\rangle=|U 0\rangle_{N S N S}+\epsilon|U 0\rangle_{R R},
$$

where $\epsilon= \pm$ distinguishes the brane from the anti-brane, and $|U 0\rangle_{N S N S}$ and $|U 0\rangle_{R R}$ are the GSO-invariant combinations in (4) and (5), respectively. The corresponding BPS state in the heterotic theory is a Kaluza-Klein excitation $\left(N_{L}=1\right)$ with momentum

$$
P_{L}=\left(0^{16} ; 0^{3}, \epsilon / R_{h 4}\right), \quad P_{R}=\left(0^{3}, \epsilon / R_{h 4}\right) .
$$

It can be shown (see [21] for details) that the masses of the two states agree.

Fractional BPS D-particles. The orbifold theory also contains a 'fractional' D-particle that is stuck at one of the fixed planes [23]. In the blow-up of the orbifold to a smooth K3, this state corresponds to a D2-brane which wraps a supersymmetric cycle [24]. In the orbifold limit the area of this cycle vanishes, but the corresponding state is not massless, due to a non-vanishing 2-form flux $B$ through the cycle [25]. In fact $B=\frac{1}{2}$, and the resulting state carries one unit of twisted-sector charge coming from the membrane itself, and one half unit of D-particle charge coming from the D2-brane worldvolume action term $\int \mathrm{d}^{3} \sigma C_{R R}^{(1)} \wedge\left(F^{(2)}+B^{(2)}\right)$. The corresponding boundary state is of the form

$\left|D 0_{f} ; \epsilon_{1}, \epsilon_{2}\right\rangle=\frac{1}{2}\left[\left(|U 0\rangle_{N S N S}+\epsilon_{1}|U 0\rangle_{R R}\right)+\epsilon_{2}\left(|T 0\rangle_{N S N S}+\epsilon_{1}|T 0\rangle_{R R}\right)\right]$,

where $|U 0\rangle_{N S N S}$ and $|U 0\rangle_{R R}$ are the same states that appeared in (11), and $|T 0\rangle_{N S N S}$ and $|T 0\rangle_{R R}$ lie in the twisted NS-NS and twisted R-R sectors, respectively. Here $\epsilon_{1}= \pm 1$ and 
$\epsilon_{1} \epsilon_{2}= \pm 1$ determine the sign of the bulk and the twisted charges of the state, respectively. As there are 16 fixed planes, there are 64 such states altogether.

The corresponding states in the heterotic theory carry momentum

$P_{L}=\left(\epsilon_{1} \epsilon_{2}\left(0^{2 n}, 1, \pm 1,0^{14-2 n}\right) ; 0^{3}, \epsilon_{1} /\left(2 R_{h 4}\right)\right), \quad P_{R}=\left(0^{3}, \epsilon_{1} /\left(2 R_{h 4}\right)\right)$.

The 16 vectors $\left(0^{2 n}, 1, \pm 1,0^{14-2 n}\right)$, with $n=1, \ldots, 8$, are in one-to-one correspondence with the 16 fixed planes in the IIA orbifold. For each such vector there are four different heterotic states. These states carry half the charge of the states in (12) with respect to the $U(1)$ that is associated with the KK momentum, and their mass is therefore also half of that of the states in (12). This is mirrored in the IIA theory, where the coefficients of the boundary state (13) in the untwisted NS-NS and untwisted R-R sector (from which the mass and charge can be read off) are half of those in (11).

\subsection{Non-BPS states}

The simplest stable non-BPS state in the heterotic theory has momentum

$$
P_{L}=\left(0^{2 n}, 2,0^{15-2 n} ; 0^{4}\right), \quad P_{R}=\left(0^{4}\right) .
$$

Level matching requires that $N_{R}-c_{R}=1$, and the state is therefore not BPS [26]. The mass of this state is $M_{h}=2 \sqrt{2} \dagger$, and it is charged under precisely two of the $16 U(1)$ 's that are associated with the 16 fixed planes in the dual type IIA theory, and uncharged with respect to any of the other $U$ (1)'s. More generally, for each pair of fixed planes, there exist four non-BPS states that carry charge \pm 1 with respect to the two corresponding $U(1)$ 's, but are uncharged with respect to any other $U(1)$.

The above state carries the same charges as two BPS states with charges

$$
\begin{aligned}
& P_{L}^{(1)}=\left(0^{2 n}, 1,1,0^{14-2 n} ; 0^{3}, 1 /\left(2 R_{h 4}\right)\right), \quad P_{R}^{(1)}=\left(0^{3}, 1 /\left(2 R_{h 4}\right)\right), \\
& P_{L}^{(2)}=\left(0^{2 n}, 1,-1,0^{14-2 n} ; 0^{3},-1 /\left(2 R_{h 4}\right)\right), \quad P_{R}^{(2)}=\left(0^{3},-1 /\left(2 R_{h 4}\right)\right) .
\end{aligned}
$$

Each of the two BPS states in (16) has mass $1 /\left(2 R_{h 4}\right)$, and the decay of (15) into (16) is energetically forbidden provided that $R_{h 4}<1 /(2 \sqrt{2})$. Similarly, we can analyse the other decay channels, and we find that (15) is stable provided that

$$
R_{h j}<\frac{1}{2 \sqrt{2}} \quad j=1,2,3,4 .
$$

As we have seen above, the two BPS states in (16) correspond, in the dual type IIA theory, to two fractional BPS D-particles that are localized at different fixed planes, and that have opposite bulk charge. The non-BPS state (15) therefore corresponds to a non-BPS D-string that stretches between a pair of fixed planes; in terms of boundary states this non-BPS state can be described as

$\left|D 1_{\text {nonbps }} ; \theta, \epsilon\right\rangle=\frac{1}{\sqrt{2}}\left[|U 1 ; \theta\rangle_{N S N S}+\frac{\epsilon}{\sqrt{2}}\left(|T 1 ; 1\rangle_{R R}+\mathrm{e}^{\mathrm{i} \theta}|T 2 ; 2\rangle_{R R}\right)\right]$.

Here $\theta=0, \pi$ describes the Wilson line on the D-string, and $|U 1 ; \theta\rangle_{N S N S}$ is defined by

$$
|U 1 ; \theta\rangle_{N S N S}=\sum_{w} \mathrm{e}^{\mathrm{i} \theta w}|U 1 ; w\rangle_{N S N S},
$$

$\dagger$ In our conventions, $\alpha_{h}^{\prime}=\frac{1}{2}$.

$\ddagger$ This state has also been independently constructed by Sen [7]. 
where $w$ denotes the winding number along the direction of the $\mathrm{D}$-string. The two states in the twisted R-R sector $|T 1 ; 1\rangle_{R R}$ and $|T 1 ; 2\rangle_{R R}$ are localized at either end of the D-string (so that the D-string stretches between two fixed planes). Using standard techniques $[4,16]$, one can easily check that each of the boundary state components is invariant under the GSO and orbifold projections. Furthermore, the spectrum of open strings beginning and ending on the same D-string can be obtained, as usual, from the cylinder amplitude of the above boundary state using worldsheet duality; for a suitable normalization of the different components, this leads to

$$
[N S-R] \frac{1}{4}\left(1+(-1)^{F} \mathcal{I}_{4}\right)\left(1+(-1)^{F} \mathcal{I}_{4}^{\prime}\right),
$$

where $\mathcal{I}_{4}^{\prime}$ is the same as $\mathcal{I}_{4}$, except that it acts on $x^{4}$ as $x^{4} \rightarrow 2 \pi R_{A 4}-x^{4}$. The boundary state (18) therefore satisfies the consistency condition (b). Sen has also argued $[1,6]$ that (c) is satisfied.

For each pair of fixed planes, there are four such D-strings that carry charge \pm 1 (depending on the four choices of $\theta=0, \pi$ and $\epsilon= \pm 1$ ) with respect to the two twisted sector $U(1)$ 's associated with the two fixed planes. These charges are of the same magnitude as those of the fractional D-particles since the ground state of $|T 1\rangle_{R R}$ is the same as that of $|T 0\rangle_{R R}$ in (13). Furthermore, it follows from (20) that the D-strings have 16 (rather than eight) fermionic zero modes and therefore transform in long multiplets of the $D=6, \mathcal{N}=(1,1)$ supersymmetry algebra. These states, therefore, have exactly the correct properties to correspond to the above non-BPS states of the heterotic theory.

The D-string that corresponds to the state (15) stretches along the $x^{4}$ coordinate in type IIA and it is stable provided that

$$
R_{A 4}<\sqrt{2} \quad \text { and } \quad R_{A j}>\frac{1}{\sqrt{2}}, \quad j=1,2,3 .
$$

Indeed, if the former inequality is violated, the open string spectrum in (20) contains a tachyon of unit KK momentum in the $x^{4}$-direction; if the latter inequality is violated, the spectrum contains a tachyon of unit winding in the $x^{1}-, x^{2}$ - or $x^{3}$-direction. These inequalities can also be determined by comparing the mass of the D-string with that of a pair of fractional BPS D-particles in the former case, and a pair of fractional BPS D2-branes in the latter.

The domains of stability (17) and (21) are qualitatively related by the duality map (9). Since the states in question are not BPS, the masses are not protected from quantum corrections, and one should not expect that the regimes of stability match exactly.

\subsection{T-duality}

T-duality relates the non-BPS D-string (18) of the type IIA orbifold to a non-BPS D-particle of type IIB on $T^{4} /(-1)^{F_{L}} \mathcal{I}_{4}[4,17]$. (Under S-duality, this orbifold is related to the orientifold of type IIB on $T^{4}$ by $\Omega \mathcal{I}_{4}$ [3] and the non-BPS D-particle corresponds to the first excited state of the string that stretches between the D5-brane and its mirror $[4,17]$.) As we have seen above, the non-BPS D-string can decay into two BPS D-particles that sit at opposite ends of the D-string and carry opposite bulk charge. Under T-duality, this configuration corresponds to a BPS D-string and an anti-D-string that carry a relative Wilson line. The above analysis, therefore, gives support for the claim that the non-BPS D-particle can indeed be described in terms of a brane-anti-brane pair [4]. 


\subsection{Other non-BPS states}

The heterotic string theory also contains stable non-BPS states that do not correspond to Dbranes in the IIA orbifold. The simplest examples are the states that transform in the spinor representation of $S O(32)$. In $D=10$ these states have been identified with a $\mathbb{Z}_{2}$-valued nonBPS D-particle in the dual type I string [5, 6, 8]. The sequence of duality transformations that relate the heterotic string on $T^{4}$ to type IIA on K3 (7) suggests that in six dimensions these states correspond to a non-BPS (non-Dirichlet) 4-brane, which may be understood as a bound state of an NS5-brane and an anti-NS5-brane.

One can also compare non-BPS states that are not necessarily stable. For example, the heterotic theory contains states that are charged with respect to a single $U(1)$ associated with one fixed plane in the dual IIA orbifold, and are uncharged with respect to any other $U(1)$; one such state is of the form

$$
P_{L}=\left(2,2,0^{16} ; 0^{4}\right), \quad P_{R}=\left(0^{4}\right) .
$$

The mass of this state is $M_{h}=2 \sqrt{6}$, and it is actually unstable in the heterotic theory $\dagger$. Indeed, (22) can decay into four BPS states of the form

$P_{L}^{(1)}=\left(1,0,1,0^{13} ; 0,0,1 /\left(2 R_{h 3}\right), 0\right)$

$$
\begin{aligned}
& P_{R}^{(1)}=\left(0,0,1 /\left(2 R_{h 3}\right), 0\right) \\
& P_{R}^{(1)}=\left(0,0,-1 /\left(2 R_{h 3}\right), 0\right) \\
& P_{R}^{(3)}=\left(0,0,1 /\left(2 R_{h 3}\right), 0\right) \\
& P_{R}^{(4)}=\left(0,0,-1 /\left(2 R_{h 3}\right), 0\right) .
\end{aligned}
$$$$
P_{L}^{(3)}=\left(0,1,0,1,0^{12} ; 0,0,1 /\left(2 R_{h 3}\right), 0\right)
$$$$
P_{L}^{(4)}=\left(0,1,0,-1,0^{12} ; 0,0,-1 /\left(2 R_{h 3}\right), 0\right)
$$

The mass of each of these states is $1 /\left(2 R_{h 3}\right)$, and this decay process is energetically forbidden provided that

$$
R_{h 3}<\frac{1}{\sqrt{6}}
$$

However, equation (22) can also decay into four winding BPS states whose left-moving (shifted) momenta are given by

$P_{L}^{(1)}=\left(\frac{1}{2}, \frac{1}{2}, 0,0,-\frac{1}{2}, \frac{1}{2}, 0,0, \frac{1}{2},-\frac{1}{2}, 0,0, \frac{1}{2}, \frac{1}{2}, 0,0 ; 0,0, R_{h 3}, 0\right)$

$P_{L}^{(2)}=\left(\frac{1}{2}, \frac{1}{2}, 0,0, \frac{1}{2},-\frac{1}{2}, 0,0,-\frac{1}{2}, \frac{1}{2}, 0,0, \frac{1}{2}, \frac{1}{2}, 0,0 ; 0,0,-R_{h 3}, 0\right)$

$P_{L}^{(3)}=\left(\frac{1}{2}, \frac{1}{2}, 0,0,-\frac{1}{2}, \frac{1}{2}, 0,0,-\frac{1}{2}, \frac{1}{2}, 0,0,-\frac{1}{2},-\frac{1}{2}, 0,0 ; 0,0, R_{h 3}, 0\right)$

$P_{L}^{(4)}=\left(\frac{1}{2}, \frac{1}{2}, 0,0, \frac{1}{2},-\frac{1}{2}, 0,0, \frac{1}{2},-\frac{1}{2}, 0,0,-\frac{1}{2},-\frac{1}{2}, 0,0 ; 0,0,-R_{h 3}, 0\right)$,

where the corresponding right-moving momenta again equal the last four entries. Each of these has mass $2 R_{h 3}$, so this decay process is forbidden when

$$
R_{h 3}>\frac{\sqrt{6}}{4}=\frac{3}{2 \sqrt{6}} \text {. }
$$

It therefore follows that the non-BPS state is unstable for all values of $R_{h 3}$.

This is actually mirrored in the dual type IIA theory, where (22) corresponds to a state that has the same charges as two fractional BPS D-particles that are located at the same fixed plane but carry opposite bulk charge. As was shown in [21], the theory does not contain a stable D-brane with these charges. Furthermore, the possible bound state of the two D-particles does not exist, since a careful analysis of the potential demonstrates that the interaction is always repulsive [27].

$\dagger$ The following discussion corrects the discussion of [21], where winding states were not considered. 


\section{Conclusions}

We have demonstrated how non-BPS D-branes can be used to probe string dualities beyond the BPS spectrum. For the case of the heterotic string on $T^{4}$ and type IIA on K3 that we have analysed in detail, both sides are quantitatively under control and one can compare the stability of the different non-BPS states. We have found that the domains of stability are qualitatively related by the duality map. It would be interesting to analyse these non-BPS states at more generic points in the moduli space of $\mathrm{K} 3$; first steps in this direction have recently been taken in $[7,28]$.

The techniques that we have described here should shed further light on the dualities that have been proposed for some non-supersymmetric theories, in particular for the models considered by Kachru et al [29] and Harvey [30] (see also [31]).

\section{Acknowledgments}

We thank Ashoke Sen for useful conversations. OB is supported in part by the DOE under grant no DE-FG03-92-ER 40701. MRG is supported by a College Lectureship of Fitzwilliam College, Cambridge.

\section{References}

[1] Sen A 1999 Non-BPS states and branes in string theory Preprint hep-th/9904207

[2] Lerda A and Russo R 1999 Stable non-BPS states in string theory: a pedagogical review Preprint hep-th/9905006

[3] Sen A 1998 J. High Energy Phys. JHEP06(1998)007 (Sen A 1998 Stable non-BPS states in string theory Preprint hep-th/9803194)

[4] Sen A J. High Energy Phys. JHEP08(1998)010 (Sen A 1998 Stable non-BPS bound states of BPS D-branes Preprint hep-th/9805019)

[5] Sen A 1998 J. High Energy Phys. JHEP09(1998)023 (Sen A 1998 SO(32) spinors of type I and other solitons on brane-antibrane pair Preprint hep-th/9808141)

[6] Sen A 1998 J. High Energy Phys. JHEP10(1998)021 (Sen A 1998 Type I D-particle and its interactions Preprint hep-th/9809111)

[7] Sen A 1998 J. High Energy Phys. JHEP12(1998)021 (Sen A 1998 BPS D-branes on non-supersymmetric cycles Preprint hep-th/9812031)

[8] Witten E 1998 J. High Energy Phys. JHEP12(1998)019 (Witten E 1998 D-branes and K-theory Preprint hep-th/9810188)

[9] Horava P 1998 Adv. Theor. Math. Phys. 21373 (Horava P 1998 Type IIA D-branes, K-theory and matrix theory Preprint hep-th/9812135)

[10] Gukov S 1999 K-Theory, reality and orientifolds Preprint hep-th/9901042

[11] Bergman O, Gimon E G and Horava P 1999 J. High Energy Phys. JHEP04(1999)010 (Bergman O, Gimon E G and Horava P 1999 Brane transfer operations and T-duality of non-BPS states Preprint hep-th/9902160)

[12] Polchinski J and Cai Y 1988 Consistency of open superstring theories Nucl. Phys. B 29691

[13] Callan C G, Lovelace C, Nappi C R and Yost S A 1988 Loop corrections to superstring equations of motion Nucl. Phys. B 308221

[14] Li M 1996 Nucl. Phys. B 460351

(Li M 1995 Boundary states of D-branes and dy-strings Preprint hep-th/9510161)

[15] Green M B and Gutperle M 1996 Nucl. Phys. B 476484

(Green M B and Gutperle M 1996 Light-cone supersymmetry and D-branes Preprint hep-th/9604091)

[16] Bergman O and Gaberdiel M R 1997 Nucl. Phys. B 499183

(Bergman O and Gaberdiel M R 1997 A non-supersymmetric open string theory and S-duality Preprint hepth/9701137)

[17] Bergman O and Gaberdiel M R 1998 Phys. Lett. B 441133

(Bergman O and Gaberdiel M R 1998 Stable non-BPS D-particles Preprint hep-th/9806155) 
[18] Blum J D and Dienes K R 1997 Phys. Lett. B 414260

(Blum J D and Dienes K R 1997 Duality without supersymmetry: the case of the $S O(16) \times S O(16)$ string Preprint hep-th/9707148)

Blum J D and Dienes K R 1998 Nucl. Phys. B 51683

(Blum J D and Dienes K R 1997 Strong/weak coupling duality relations for non-supersymmetric string theories Preprint hep-th/9707160)

[19] Bergman O and Gaberdiel M R 1999 J. High Energy Phys. JHEP07(1999)022

(Bergman O and Gaberdiel M R 1999 Dualities of type 0 strings Preprint hep-th/9906055)

[20] Blumenhagen R and Kumar A 1999 Phys. Lett. B 46446

(Blumenhagen R and Kumar A 1999 A note on orientifolds and dualities of type 0B string theory Preprint hep-th/9906234)

[21] Bergman O and Gaberdiel M R 1999 J. High Energy Phys. JHEP03(1998)013

(Bergman O and Gaberdiel M R 1999 Non-BPS states in heterotic-type IIA duality Preprint hep-th/9901014)

[22] Witten E 1995 Nucl. Phys. B 44385

(Witten E 1995 String theory dynamics in various dimensions Preprint hep-th/9503124)

[23] Douglas M and Moore G 1996 D-branes, quivers and ALE instantons Preprint hep-th/9603167

[24] Douglas M 1997 J. High Energy Phys. JHEP07(1997)004 07004

(Douglas M 1997 Enhanced gauge symmetry in M(atrix) theory Preprint hep-th/9612126)

Diaconescu D, Douglas M and Gomis J 1998 J. High Energy Phys. JHEP02(1998)013

(Diaconescu D, Douglas M and Gomis J 1997 Fractional branes and wrapped branes Preprint hep-th/9712230)

Berenstein D and Corrado R 1998 Nucl. Phys. B 529225

(Berenstein D and Corrado R 1998 Matrix theory on ALE spaces and wrapped membranes Preprint hepth/9803048)

[25] Aspinwall P S 1995 Phys. Lett. B 357329

(Aspinwall P S 1995 Enhanced gauge symmetries and K3 surfaces Preprint hep-th/9507012)

[26] Dabholkar A and Harvey J 1989 Nonrenormalization of the superstring tension Phys. Rev. Lett. 63478

[27] Gaberdiel M R and Sen A 1999 J. High Energy Phys. JHEP11(1999)008

(Gaberdiel M R and Sen A 1999 Non-supersymmetric D-Brane configurations with bose-fermi degenerate open string spectrum Preprint hep-th/9908060)

[28] Majumder J and Sen A 1999 J. High Energy Phys. JHEP09(1999)004

(Majumder J and Sen A 1999 Blowing up D-branes on non-supersymmetric cycles Preprint hep-th/9906109)

[29] Kachru S, Kumar J and Silverstein E 1999 Phys. Rev. D 59106004

(Kachru S, Kumar J and Silverstein E 1998 Vacuum energy cancellation in a non-supersymmetric string Preprint hep-th/9807076)

[30] Harvey J A 1999 Phys. Rev. D 59026002

(Harvey J A 1998 String duality and non-supersymmetric strings Preprint hep-th/9807213)

[31] Körs B 1999 J. High Energy Phys. JHEP11(1999)028

(Körs B 1999 D-brane spectra of nonsupersymmetric, asymmetric orbifolds and nonperturbative contributions to the cosmological constant Preprint hep-th/9907007) 